\title{
QUANTITATIVE CIRCULATORY MEASUREMENTS IN BRANCH RETINAL VESSEL OCCLUSION
}

\author{
NAOKI FUJIO ${ }^{1,2}$, GILBERT T. FEKE ${ }^{1}$, HIRONOBU OGASAWARA ${ }^{1,2}$, DOUGLAS G. GOGER ${ }^{1}$, \\ AKITOSHI YOSHIDA' ${ }^{1,2}$ and J. WALLACE McMEEL ${ }^{\prime}$ \\ Boston, Massachusetts and Asahikawa, Japan
}

\begin{abstract}
SUMMARY
We used the laser Doppler technique to quantify retinal circulatory abnormalities in three patients with branch retinal artery occlusion and in two patients with branch retinal vein occlusion, each of whom had shown delayed filling on fluorescein angiography. Blood flow in occluded arteries was $\mathbf{4 0 - 5 0 \%}$ lower than in non-occluded arteries of the same branching order. In the patients with branch retinal vein occlusion blood flow in arteries supplying the affected regions was $\mathbf{8 0 - 9 0 \%}$ lower than in arteries supplying the unaffected regions. One vein occlusion patient was measured serially over 18 months. Blood flow increased from 4 to $12 \mu \mathrm{l} / \mathrm{min}$ in the artery supplying the affected region in this patient. The results demonstrate that retinal circulatory abnormalities can be evaluated quantitatively during the course of retinal vascular occlusive disease, and suggest that the efficacy of therapy can be monitored using our techniques.
\end{abstract}

Aside from diabetic retinopathy, branch retinal vessel occlusion is the most common retinal vascular problem. The natural history of both branch retinal vein occlusion and branch retinal artery occlusion and the effects of different treatment modalities on the evolution of these disorders have been clinically well documented. ${ }^{1-4}$ Fluorescein angiography has been used to investigate the retinal circulatory changes that accompany the disease process. Initial studies aimed at quantifying these retinal circulatory changes were reported by Hill ${ }^{5}$ and David et $a l .{ }^{6}$ Fluorescein angiography, however, provides only a semi-quantitative measure of the circulatory impairment during the disease process. The purpose of our study was to quantify retinal circulatory abnormalities in patients with branch retinal vessel occlusions using the laser Doppler technique and to compare the results with fluorescein angiography.

From: 'The Schepens Eye Research Institute and Harvard Medical School, Boston, Massachusetts, USA; ${ }^{2}$ Department of Ophthalmology, Asahikawa Medical College, Asahikawa, Japan.

Correspondence to: Gilbert T. Feke, PhD, The Schepens Eye Research Institute, 20 Staniford Street, Boston, MA 02114, USA.

\section{PATIENTS AND METHODS}

Three patients with branch retinal artery occlusion and two patients with branch retinal vein occlusion were subjects for the study. One of the vein occlusion patients (patient 1) was studied serially over a period of 18 months. Each patient received a complete eye examination consisting of best corrected visual acuity, intraocular pressure, indirect ophthalmoscopy, slit lamp biomicroscopy and fundus colour photography. Monochromatic $(575 \mathrm{~nm})$ fundus photographs were taken of the eye chosen for study.

The procedures performed in the study followed the tenets of the Helsinki Declaration, and were approved by the Schepens Eye Research Institute Human Studies Committee. Written informed consent was obtained from each patient.

Patient 1 was a 67-year-old woman who had been diagnosed with a branch retinal vein occlusion in her right eye 3 months prior to initial laser Doppler testing. The patient had no retinal symptoms, complaining only of a dry eye condition. On the day of initial laser Doppler testing, best corrected visual acuity was 20/30 in the right eye and $20 / 25$ in the left eye, intraocular pressure was $14 \mathrm{mmHg}$ in the right eye and $16 \mathrm{mmHg}$ in the left, and the anterior segments of both eyes were normal. Indirect ophthalmoscopy of the right eye showed flame-shaped retinal haemorrhages along the inferotemporal retinal vein. Follow-up laser Doppler measurements were obtained 4, 8, 12 and 18 months after initial testing. Fluorescein angiograms of the right eye were obtained 4 and 18 months after initial testing. Clinically, during this period visual acuity and intraocular pressure remained stable. There was a decrease in the number and extent of retinal haemorrhages, and the appearance of well-developed collaterals.

Patient 2 was a 69-year-old man who had noticed a sudden onset of blurring in the left eye 2 weeks prior to laser Doppler testing. One week later, a local ophthalmologist obtained a fluorescein angiogram and diagnosed a branch retinal vein occlusion. The patient was referred for further 
evaluation. On the day of laser Doppler testing, best corrected visual acuity was 20/40 in the right eye and 20/50 in the left. Intraocular pressure was $18 \mathrm{mmHg}$ in the right eye and $20 \mathrm{mmHg}$ in the left. Indirect ophthalmoscopy of the left eye showed scattered, flame-shaped haemorrhages along the superotemporal vein.

Patient 3 was a 63 -year-old woman who had noticed a sudden onset of blurring in the right eye. The next day, a local ophthalmologist examined her and diagnosed a branch retinal artery occlusion in the right eye and referred her for further evaluation including a fluorescein angiogram. The next day, at the time of laser Doppler testing, best corrected visual acuity was 20/50 in the right eye and $20 / 30$ in the left. Intraocular pressure was $10 \mathrm{mmHg}$ in the right eye and $15 \mathrm{mmHg}$ in the left. Indirect ophthalmoscopy of the right eye showed an embolus along the superotemporal arcade and an oval-shaped area of retinal whitening that corresponded to the territory of the obstructed arteriole.

Patient 4 was a 61-year-old woman who had complained of cloudy vision in the right eye 1 week prior to laser Doppler testing. Indirect ophthalmoscopy revealed a superior branch retinal artery occlusion of the right eye. A fluorescein angiogram was obtained. On the day of laser Doppler testing, best corrected visual acuity was 20/30 in the right eye and 20/20 in the left, intraocular pressure was $23 \mathrm{mmHg}$ in the right eye and $28 \mathrm{mmHg}$ in the left eye. Indirect ophthalmoscopy of the right eye showed whitening of the upper half of the retina and an embolus was seen just above the base of the superior retinal artery.

Patient 5 was a 63 -year-old man who experienced transient sudden losses of vision in the left eye 5 weeks prior to laser Doppler testing. He was referred by a local ophthalmologist for further evaluation. On the day of laser Doppler testing, best corrected visual acuity was 20/20 in both eyes, intraocular pressure was $22 \mathrm{mmHg}$ in the right eye and $21 \mathrm{mmHg}$ in the left eye. Indirect ophthalmoscopy of both eyes was unremarkable. However, a fluorescein angiogram of the left eye suggested a diagnosis of a superior temporal arterial occlusion.

The methodology of our application of the laser Doppler technique to measurement of centerline blood velocity in retinal arteries has been described previously. ${ }^{7.8}$ Our projection micrometry technique for measurement of arterial diameters using monochromatic photographs has also been previously described. ${ }^{9}$

Blood flow rate in an individual retinal artery was calculated as Flow $=\bar{V} \times$ Area/2, where $\bar{V}$ is the time average of the centreline blood velocity during the cardiac cycle, and Area is the cross-sectional area of the retinal artery at the laser Doppler measurement site. ${ }^{10}$ The area was calculated from the arterial diameter assuming a circular cross-section.

In the two patients with branch retinal vein occlusion laser Doppler measurements were obtained from the artery supplying the affected region of the retina as well as from a comparable artery supplying an unaffected region of the retina in the same eye. In patient 3 measurements were made at three arterial sites in the vicinity of the obstructed arteriole and at a fourth site in an artery supplying an unaffected region of the retina in the same eye. In the other two patients with branch retinal artery occlusion measurements were obtained from the affected artery and from an unaffected artery in the same eye.

\section{RESULTS}

Fig. 1 shows a pair of arteriovenous phase fluorescein angiograms of the right eye of patient 1 , obtained 4 months and 18 months after initial laser Doppler testing. Also shown is the blood flow rate measured using the laser Doppler technique in the inferotemporal retinal artery supplying the region of the retina affected by the inferotemporal venous occlusion, and the flow rate in the superotemporal artery supplying the unaffected region. The angiogram taken at 4 months shows delayed filling of the occluded vein in the inferotemporal quadrant. Multiple capillary dilatations are present in the macula. The angiogram taken at 18 months shows improvement in the filling of the inferotemporal vein as well as the appearance of well-developed vascular collaterals inferotemporal to the optic disc. There was a threefold increase in the blood flow rate in the inferotemporal artery during the 14 month period between the angiograms. The blood flow rate in the superotemporal artery remained stable.

Fig. 2 shows the centerline blood speeds and blood flow rates measured serially in the inferotemporal retinal artery of patient 1 . Initially and 4 months later, the blood speed measured during the diastolic phase of the cardiac cycle was essentially zero. At these visits, the blood speed measured during the systolic phase of the cardiac cycle was $2-3 \mathrm{~cm} / \mathrm{s}$. With the development of the collateral vessels seen in the angiogram at 18 months, the blood speed measured during diastole increased to $2 \mathrm{~cm} / \mathrm{s}$, and the blood speed measured during systole increased to $5-6 \mathrm{~cm} / \mathrm{s}$. As also shown in Fig. 2, the corresponding blood flow rate increased from 4 to $12 \mu \mathrm{l} /$ min during the 18-month follow-up period.

Fig. 3 is an arteriovenous phase fluorescein angiogram of the left eye of patient 2. Delayed filling of the occluded vein in the superotemporal quadrant is seen. Centerline blood speed in the superotemporal artery supplying the affected region of the retina ranged from $0.4 \mathrm{~cm} / \mathrm{s}$ during diastole to $1.8 \mathrm{~cm} / \mathrm{s}$ during systole. In contrast, blood speed in the inferotemporal artery supplying the unaffected region of the retina ranged from $3.8 \mathrm{~cm} / \mathrm{s}$ during diastole to $12.5 \mathrm{~cm} / \mathrm{s}$ during systole. The corresponding blood flow rates are shown in the figure.

Fig. 4 is an arteriovenous phase fluorescein angiogram of the right eye of patient 3 . There is an obstructive plaque just past the first bifurcation in the superotemporal artery and delayed filling of the arterioles and venules in the more temporal region of the superotemporal network. Laser Doppler measurements were obtained from the superotemporal artery before the bifurcation, in the branch with the obstruction, and in the branch without the obstruction. A fourth measurement was obtained from the 

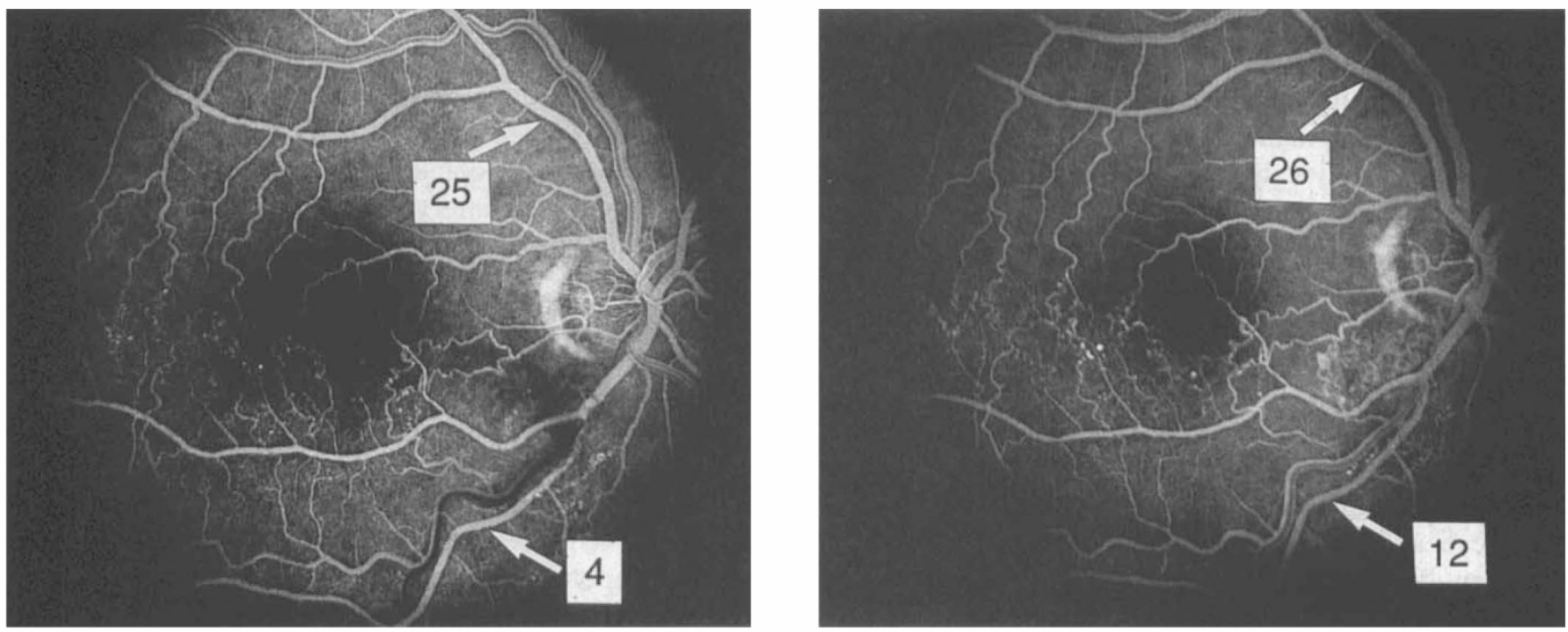

Fig. 1. Arteriovenous phase fluorescein angiograms of the right eve of patient 1, 4 months (left) and 18 months (right) after initial laser Doppler testing. The blood flow rates $(\mu \mathrm{l} / \mathrm{min})$ in the superotemporal and inferotemporal arteries are shown.

unaffected inferotemporal artery. The blood flow rates measured at these sites are shown in the figure. The blood flow in the superotemporal artery is markedly lower than in the inferotemporal artery, and the blood flow in the occluded branch of the superotemporal artery is much lower than in the unaffected branch.

Fig. 5 is an arteriovenous phase fluorescein angiogram of the right eye of patient 4 . There is delayed filling of the veins in the superotemporal quadrant. Laser Doppler measurements were obtained from the superotemporal and inferotemporal retinal arteries. The blood flow was markedly lower in the superotemporal artery.

Fig. 6 is an arteriovenous phase fluorescein angiogram of the left eye of patient 5 , who had been diagnosed with a superotemporal arterial occlusion. The angiogram shows delayed filling of the veins in the superotemporal quadrant. The laser Doppler blood flow rate measured in the

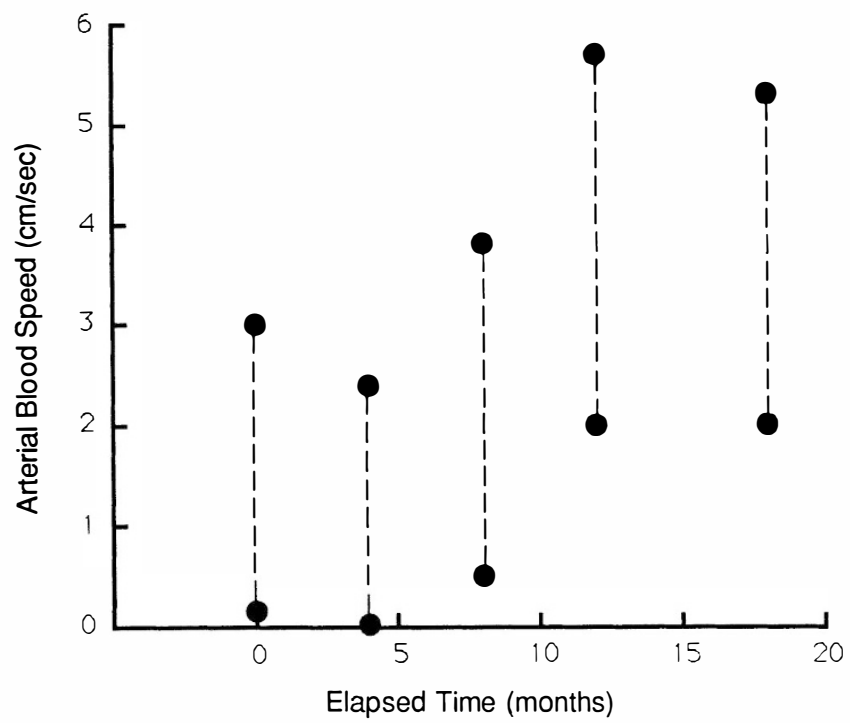

superotemporal artery is only one-half of that measured in the unaffected inferotemporal artery.

\section{DISCUSSION}

The results show that the laser Doppler technique can be used to quantify the local retinal blood flow abnormalities that are present in patients with branch retinal vessel occlusion. In each patient studied, fluorescein angiography provided a semi-quantitative indication of the circulatory impairment in terms of the finding of delayed filling of the veins draining the affected regions of the retina. The laser Doppler results showed that blood flow in the affected arteries of the patients with branch retinal artery occlusion was $40-50 \%$ lower than in unaffected arteries of the same branching order. In the patients with branch retinal vein occlusion, blood flow in arteries

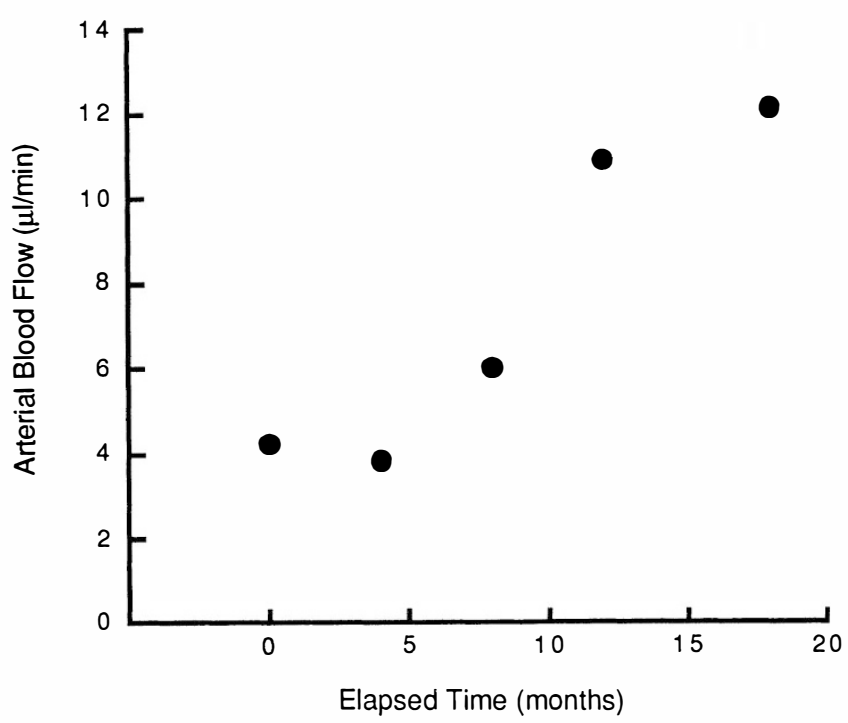

Fig. 2. The centreline blood speeds (left) and the blood flow rates (right) measured initially and 4, 8, 12 and 18 months later in the inferotemporal retinal artery of patient 1 . The lower set points on the left show's the blood speeds measured during the diastolic phase of the cardiac cycle; the upper set shows the speeds measured during the systolic phase. 


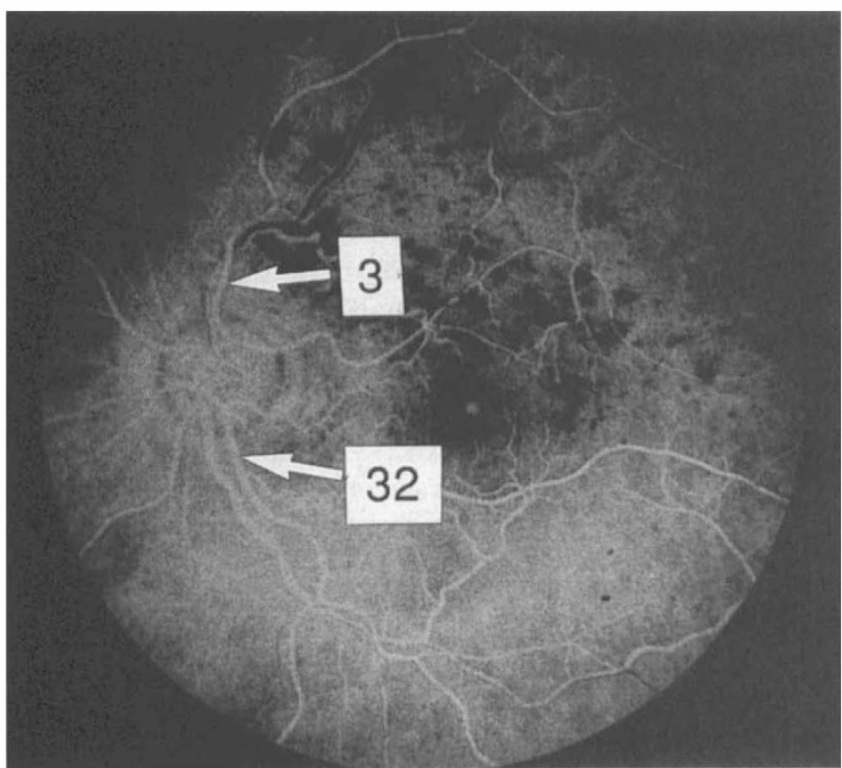

Fig. 3. Arteriovenous phase fluorescein angiogram of the left eye of patient 2 . The blood flow rates $(\mu \mathrm{l} / \mathrm{min})$ in the superotemporal and inferotemporal arteries are shown.

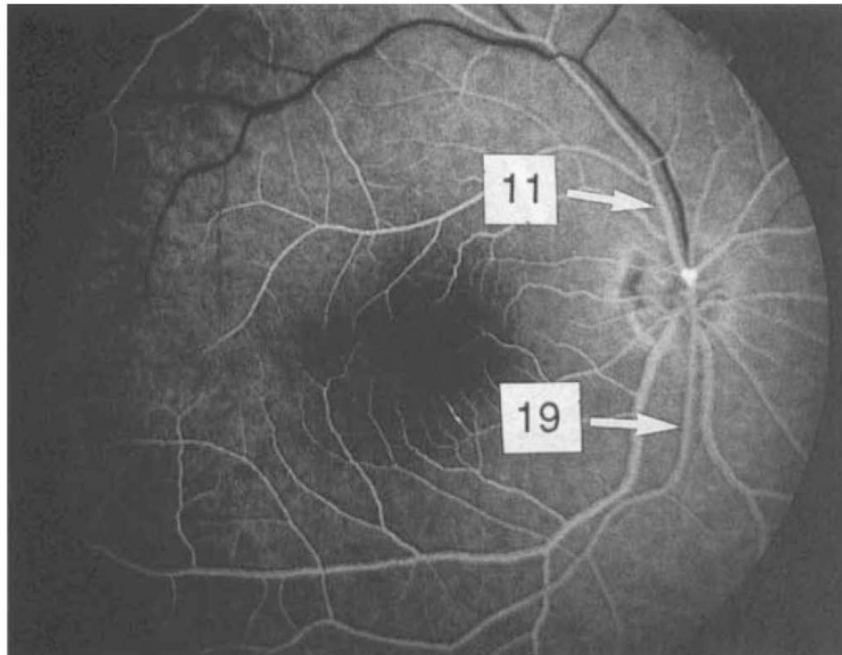

Fig. 5. Arteriovenous phase fluorescein angiogram of the right eye of patient 4 . The blood flow rates $(\mu \mathrm{l} / \mathrm{min})$ in the superotemporal and inferotemporal arteries are shown.

supplying the affected regions was 80-90\% lower than in arteries supplying the unaffected regions.

The laser Doppler results on patient 1, who was measured serially over a period of 18 months, provided insight into the haemodynamic abnormalities associated with branch retinal vein occlusion. As shown in Fig. 2, the arterial blood speed measured during the diastolic phase of the cardiac cycle was essentially zero both at the time of the initial measurement and 4 months later. The cessation of flow during diastole is an indication that the retinal arterial diastolic blood pressure was not great enough to overcome the backpressure in the arteriovenous vascular segment created by the vein occlusion. In this circum-

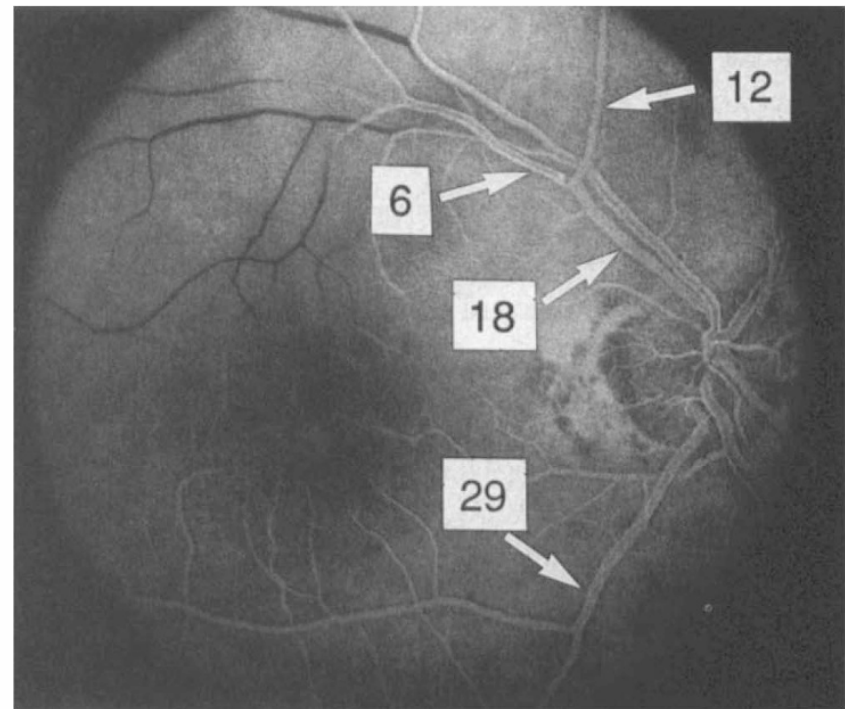

Fig. 4. Arteriovenous phase fluorescein angiogram of the right eye of patient 3 . The blood flow rates $(\mu \mathrm{l} / \mathrm{min})$ in the supertemporal artery before the bifurcation, in the branch with the obstruction, in the branch without the obstruction, and in the inferotemporal artery are shown.

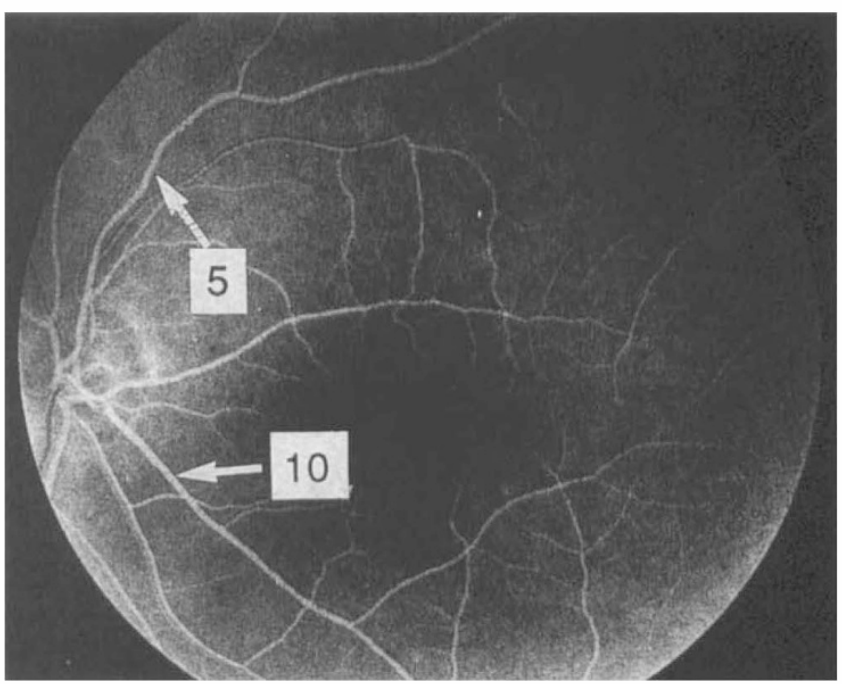

Fig. 6. Arteriovenous phase fluorescein angiogram of the left eye of patient 5 . The blood flow rates $(\mu \mathrm{l} / \mathrm{min})$ in the superotemporal and inferotemporal arteries are shown.

stance, blood flow occurred only during the systolic phase of the cardiac cycle.

The continuous improvement in arterial blood flow in patient 1 measured 8, 12 and 18 months after initial testing is most probably associated with the development of collateral vessels, which act to bypass the venous occlusion site. The time frame for the improvement in blood flow is consistent with the observation of Pieris and Hill, " who studied the development and natural history of collateral vessels in patients with branch retinal vein occlusion. The improvement in blood flow related to the development of collateral vessels has also been documented by Mihara ${ }^{12,13}$ who used videofluorescein angiography and image analy- 
sis to compare fluorescein circulation times measured in patients with relatively fresh branch retinal vein occlusions with the circulation times measured in patients with long-standing branch retinal vein occlusions.

In summary, our results demonstrate that retinal circulatory abnormalities can be evaluated quantitatively during the course of retinal vascular occlusive disease, and suggest that the efficacy of therapy can be monitored using our techniques.

This work was supported in part by USPH Grant EY01303 from the National Institutes of Health and the Massachusetts Lions Eye Research Fund. The authors wish to thank Alex E. Jalkh, MD, Sheldon M. Buzney, MD, and Jay S. Duker, MD, for referring patients for this study.

Key words: Branch retinal artery occlusion, Branch retinal vein occlusion, Laser Doppler technique, Retinal blood flow.

\section{REFERENCES}

1. Michels RG, Gass JDM. The natural course of retinal branch vein obstruction. Trans Am Acad Ophthalmol Otolaryngol 1974;78:OP166-77.

2. Patz A, Yassur Y, Fine SL, Finkelstein D, Orth DH. Branch retinal vein occlusion. Trans Am Acad Ophthalmol Otolaryngol 1977;83:OP373-8.

3. Orth DH, Patz A. Retinal branch vein occlusion. Surv Ophthalmol 1978;22:357-76.

4. Ros MA, Magargal LE, Uram M. Branch retinal-artery obstruction: a review of 201 eyes. Ann Ophthalmol 1989;21:103-7.
5. Hill DW. Fluorescein studies in retinal vascular occlusion. Br J Ophthalmol 1968;52:1-12.

6. David NJ, Gilbert DS, Gass JDM. Fluorescein angiography in retinal arterial branch obstructions. Am J Ophthalmol 1970;69:43-55.

7. Feke GT, Goger DG, Tagawa H, Delori FC. Laser Doppler technique for absolute measurement of blood speed in retinal vessels. IEEE Trans Biomed Eng 1987;34:673-80.

8. Ogasawara H, Feke GT, Yoshida A, Milbocker MT, Weiter JJ, McMeel JW. Retinal blood flow alterations associated with scleral buckling and encircling procedures. $\mathrm{Br} \mathrm{J}$ Ophthalmol 1992;76:275-9.

9. Delori FC, Fitch KA, Feke GT, Deupree DM, Weiter JJ. Evaluation of micrometric and microdensitometric methods for measuring the width of retinal vessel images on fundus photographs. Graefes' Arch Clin Exp Ophthalmol 1988; 226:393-9.

10. Feke GT, Tagawa H, Deupree DM, Goger DG, Sebag J, Weiter JJ. Blood flow in the normal human retina. Invest Ophthalmol Vis Sci 1989;30:58-65.

11. Pieris SJP, Hill DW. Collateral vessels in branch retinal vein occlusion. Trans Ophthalmol Soc UK 1982;102:178-81.

12. Mihara M. Retinal circulation in old branch retinal vein occlusion demonstrated by videofluorescence angiography and image analysis. Nippon Ganka Gakkai Zasshi 1988; 92:359-66.

13. Mihara M. Retinal circulation in fresh branch retinal vein occlusion demonstrated by videofluorescence angiography and image analysis. Nippon Ganka Gakkai Zasshi 1988;92:1242-7. 